\title{
Distribution of the number of particles in the final state of hadron-nucleus collisions*
}

\author{
Laura Dominé, Cédric Lorcé, Stéphane Munier', Simon Pekar \\ Centre de physique théorique, École polytechnique, CNRS, Université Paris-Saclay, Palaiseau, \\ France \\ E-mail: laura.domineepolytechnique.edu, \\ cedric.lorce@polytechnique.edu, stephane.munier@polytechnique.edu, \\ simon.pekar@polytechnique.edu
}

\begin{abstract}
Recently, Liou, Mueller and Munier have argued that proton-nucleus collisions at the LHC may give access to the full statistics of the event-by-event fluctuations of the gluon density in the proton. Indeed, the number of particles produced in an event in rapidity slices in the fragmentation region of the proton may, under some well-defined assumptions, be directly related to the number of gluons which have a transverse momentum larger than the nuclear saturation scale present in the proton at the time of the interaction with the nucleus. A first calculation of the probability distribution of the number of gluons in a hadron was performed, using the color dipole model. In this talk, we review this proposal, and present preliminary numerical calculations which support the analytical results obtained so far.
\end{abstract}

XXV International Workshop on Deep-Inelastic Scattering and Related Subjects

3-7 April 2017

University of Birmingham, $U K$

\footnotetext{
*This work is supported by the Agence Nationale de la Recherche under the project ANR-16-CE31-0019.

† Speaker.
} 


\section{Introduction}

The LHC has opened a new window on hadronic physics by enabling the measurement of a wealth of new observables in the collisions of protons and nuclei, at unprecedented center-of-mass energies. In particular, there is a lot of interest in trying to pin down higher moments or even the full distribution of the event-by-event fluctuations of observables, with a view to characterizing how hadrons look in individual events. For example, it has been proposed to use diffractive dissociation processes to constrain the fluctuations of the shape of the proton [1]. This contribution reports on an effort to understand the fluctuations of the integrated gluon density in the proton.

In the recent paper of Ref. [2], it has been proposed to study the event-by-event fluctuations of the multiplicity of particles produced in specific regions in rapidity in the final state of protonnucleus collisions, arguing that such an observable would be directly related to the probability distribution of the gluon density in the proton. Considering an onium instead of a proton, a calculation of the probability of particle numbers was attempted, based on the color dipole model [3, 4] to represent the QCD evolution, modified in the infrared in order to mimick confinement.

We shall review this simple picture for particle production and the corresponding calculation of the multiplicity distribution, and present preliminary numerical evaluations of the distribution of the number of particles produced in onium-nucleus collisions.

\subsection{Picture of particle production in proton-nucleus collisions}

Let us consider the collision of a proton with a large nucleus. The proton is an initially dilute object, while the nucleus is a dense object characterized by the saturation momentum $Q_{A}$ in its rest frame. The physical meaning of $Q_{A}$ is the following: To a globally colorless probe whose colored constituents carry the relative transverse momentum $k \gg Q_{A}$ (or are separated by the distance $\left.r \sim 1 / k \ll 1 / Q_{A}\right)$, the nucleus appears dilute, while a probe characterized by $k \ll Q_{A}$ is absorbed with probability one in an interaction with this nucleus [4].

The wave function of a nucleus is almost the same as the ground-state wave function of nuclear matter, whose density is essentially uniform [2]. If we look at it from the point of view of a probe at rest in a frame in which it has the rapidity $y_{0}$ (which we shall call the " $y_{0}$-frame"1), then the QCD evolution populates its Fock state with many gluons, but in an almost deterministic way since it starts already with a large number of valence quarks. Hence the state of such a nucleus is still essentially characterized by a single momentum scale (unfluctuating, i.e. identical in each event), the saturation momentum $Q_{s}\left(y_{0}\right)$, larger than $Q_{A}$ by a factor which grows exponentially with $y_{0}$.

In the $y_{0}$-frame, the proton has the rapidity $Y-y_{0}$, where $Y$ is the total rapidity of the scattering determined by the center-of-mass energy. In each event, the proton is found in a given Fock state essentially made of many gluons, produced through QCD evolution. Since the proton is a dilute object in its rest frame, when viewed at a nonzero rapidity, its content is expected to strongly fluctuate from event to event, unlike the nucleus. We choose $y_{0}$ such that there is a significant QCD evolution in the proton, but not too large, in such a way that nonlinear saturation effects can always be neglected.

The effect of the interaction of this proton with the nucleus is to filter the gluons which have a transverse momentum less than $Q_{s}\left(y_{0}\right)$ (thanks to multiple scatterings with the nucleus which

\footnotetext{
${ }^{1}$ We borrow this terminology and the notations from Ref. [5].
} 
transfer them energy to put them on-shell). These gluons go to the final state and hadronize. The other gluons essentially do not interact and go back to the vacuum at finite time. This intuitive picture is backed by QCD calculations [4].

Hence the particle multiplicity measured in the proton fragmentation region in an event is essentially proportional to the gluon number density $x G$ integrated at the scale $Q_{s}\left(y_{0}\right)$ in the corresponding realization of the QCD evolution. More quantitatively, the number $d N / d y_{2}$ of particles per unit rapidity measured around the rapidity $y_{2}=0$ in the $y_{0}$-frame reads [5]

$$
\left.\frac{d N}{d y_{2}}\right|_{y_{2} \simeq 0}=x G\left(x, Q_{s}^{2}\left(y_{0}\right)\right),
$$

where $x=e^{-\left(Y-y_{0}\right)} Q_{s}\left(y_{0}\right) / M$ with $M$ the mass of the onium. Strictly speaking, this formula is established as an equality between mean quantities (averaged over events), but we shall assume that it also holds for each event considered separately. According to this picture, measuring the distribution of the particle number in the final state of $p A$ collisions amounts to measuring the fluctuations of the gluon number density in the proton.

So far, experimental and theoretical studies of the partonic content of the proton have focused on the expectation value of parton number densities (related to total cross sections, for example of deep-inelastic scattering processes) and their second moments (related to diffractive dissociation). Hence such an observable in proton-nucleus scattering would give access to genuinely new information on the parton densities, namely their full probability distribution.

We shall now present a QCD calculation of the fluctuations of the gluon number density in a dilute hadron.

\subsection{How a hadronic state dresses at high energies: the color dipole model}

Instead of addressing proton-nucleus collisions, we study the scattering of onia (which have the advantage of being color dipoles) of initial fixed transverse size $\underline{r}_{0}$ off large (i.e. infinite transverse extent) nuclei. An onium is the simplest model of a dilute hadron, and the main features of our calculation may go over to the proton case.

In a frame in which the rapidity $y=Y-y_{0}$ of the onium is large, the Fock state is a dense system of gluons. The leading contribution to the probability of such a state (i.e. the large-y asymptotics) is obtained by calculating the splitting of the initial quark-antiquark into a state made of the $q \bar{q}$ pair supplemented with a soft gluon. The latter may split further into a denser state populated with softer and softer gluons. This is essentially the physics captured by the BalitskyFadin-Kuraev-Lipatov (BFKL) equation [4].

Taking the large number-of-color $\left(N_{c}\right)$ limit simplifies the color structure of these complicated gluonic states, and leads to the famous color dipole model [3]. The relevant Fock states may be constructed iteratively from one single elementary process: The splitting of a dipole of size $\underline{r}_{0}$ into two dipoles of size vectors $\underline{r}$ and $\underline{r}-\underline{r}_{0}$. Its rate (as the rapidity increases) is computed in perturbative QCD and reads

$$
\frac{d P}{d y}=\bar{\alpha} \frac{d^{2} \underline{r}}{2 \pi} \frac{\underline{r}_{0}^{2}}{\underline{r}^{2}\left(\underline{r}_{0}-\underline{r}\right)^{2}},
$$

where $\bar{\alpha}=\alpha_{s} N_{c} / \pi$. This branching process is represented schematically in Fig. 1, together with the shape of the dipole distribution obtained in a typical event. 


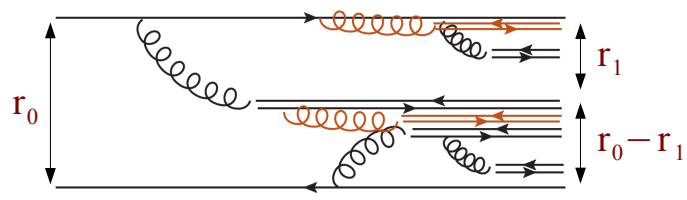

(a)

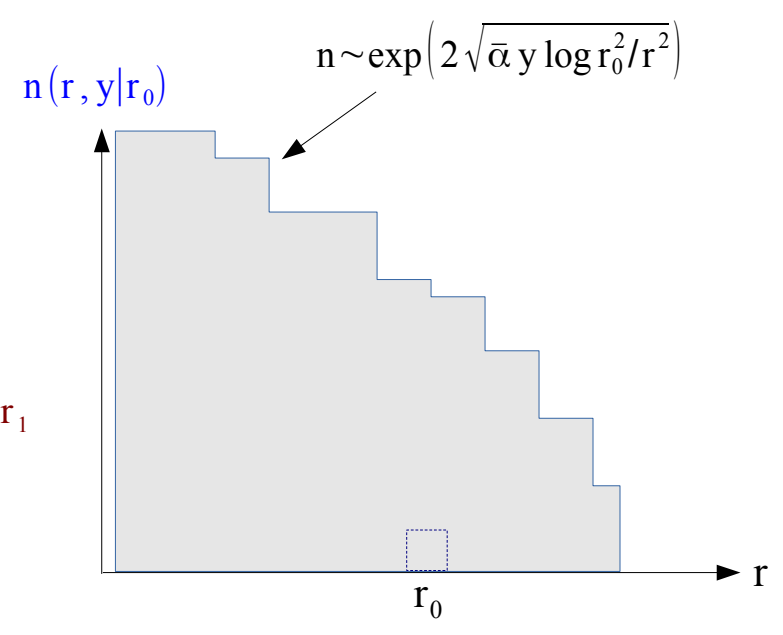

(b)

Figure 1: (a) Picture of the dressing of an onium of size $r_{0}$ boosted to rapidity $y=Y-y_{0}$ by gluonic fluctuations, represented by dipoles in the large- $N_{c}$ limit. Due to the collinear singularity in the splitting probability, many small dipoles are produced. (b) Sketch of the number of dipoles $n\left(r, y \mid r_{0}\right)$ of size larger than $r$ in an onium of size $r_{0}$ after evolution over the rapidity $y$ in a typical event, when $y \gg 1 / \bar{\alpha}$, as a function of $r$ (logarithmic scale on both axis). In the region $r \ll r_{0}, n$ follows the well-known double-logarithmic form $n \sim \exp \left(2 \sqrt{\bar{\alpha} y \ln r_{0}^{2} / r^{2}}\right)$, which is easy to prove for the mean dipole number $\bar{n}$, but which is also true for individual events provided the number of dipoles is large enough so that the law of large numbers applies.

Iterating the dipole branching process, we arrive at a random set of dipoles. Let us define $n\left(r, y \mid r_{0}\right)$ the number of dipoles of size larger than $r$ in an onium of size $r_{0}$ at rapidity $y$. The density of gluons in the onium carrying the fraction $x$ of its momentum and having transverse momentum $k$ is related to the number of dipoles $n$ in the following way [2]:

$$
x G\left(x, k^{2}\right)=\left.\frac{\partial}{\partial y}\right|_{y=\ln (1 / x)} n\left(1 / k, y \mid r_{0}\right)
$$

Strictly speaking, this relation is established in the double-logarithmic approximation, and after averages over the events have been taken. Again, we shall assume that it is actually valid for each event.

Non-perturbative physics such as confinement cannot of course be captured by the original color dipole model. Roughly speaking, confinement restricts the size of color dipoles to be less than the typical hadronic size $1 / \Lambda_{\mathrm{QCD}}$. We shall model it by multiplying the dipole splitting rate by the ad hoc cutoff function $\Theta$ :

$$
\left.\frac{d P}{d y}\right|_{\text {Eq. (1.2) }} \longrightarrow \frac{d P}{d y}=\bar{\alpha} \frac{d^{2} \underline{r}}{2 \pi} \frac{\underline{r}_{0}^{2}}{\underline{r}^{2}\left(\underline{r}_{0}-\underline{r}\right)^{2}} \Theta\left(\underline{r}, \underline{r}_{0}-\underline{r} ; R_{\mathrm{IR}}\right),
$$

chosen in such a way that $\Theta\left(\underline{r}, \underline{r}_{0}-\underline{r} ; R_{\mathrm{IR}}\right)$ tends to zero when $r \gg R_{\mathrm{IR}}$ or $\left|\underline{r}_{0}-\underline{r}\right| \gg R_{\mathrm{IR}}$ and to one when both $r$ and $\left|\underline{r}_{0}-\underline{r}\right|$ are much less than $R_{\mathrm{IR}}$. 


\section{Probability distribution of the particle multiplicity}

\subsection{Analytical insights}

Let us define $P_{n}(r, y)$ the probability to have $n$ dipoles of size larger than $1 / Q_{s}\left(y_{0}\right)$ in the Fock state at rapidity $y$. It is not difficult to write down a system of evolution equations for $P_{n}$ :

$$
\frac{\partial P_{n}\left(r_{0}, y\right)}{\partial y}=\bar{\alpha} \int \frac{d^{2} \underline{r}}{2 \pi} \frac{\underline{r}_{0}^{2}}{\underline{r}^{2}\left(\underline{r}_{0}-\underline{r}\right)^{2}} \Theta\left(\underline{r}, \underline{r}_{0}-\underline{r} ; R_{\mathrm{IR}}\right)\left[\sum_{m=1}^{n-1} P_{m}(r, y) P_{n-m}\left(\left|\underline{r}_{0}-\underline{r}\right|, y\right)-P_{n}\left(r_{0}, y\right)\right] .
$$

This infinite set of equations may be summarized by writing the evolution equation for the generating functional of the factorial moments of $P_{n}$, which turns out to coincide with the BalitskyKovchegov (BK) equation [4]. Hence, solving (2.1) is tantamount to solving the BK equation, which so far has not been achieved.

The analytical and numerical study of the fluctuations of the number of dipoles was pioneered in Ref. [6] in the context of the color dipole model without an infrared cutoff (i.e. for $\Theta=1$ ). In this case, the probability $P_{n}$ was found to exhibit a fat tail

$$
P_{n} \underset{n \gg \bar{n}}{\sim} \exp \left(-\frac{\ln ^{2} n}{4 \bar{\alpha} y}\right) .
$$

The large-multiplicity events are characterized by the presence of a large dipole, produced at the very beginning of the QCD evolution. Its size may become arbitrarily large as one selects events which have a larger and larger occupancy.

When confinement is accounted for, such large dipoles may not be produced. This results in a drastic damping of the tail of the probability distribution, which becomes exponential:

$$
P_{n} \underset{n \gg \bar{n}}{\sim} e^{-n /(c \bar{n})},
$$

where $\bar{n}$ is the mean dipole number (i.e. averaged over events) and $c$ is a constant a priori of order 1 , but which we have not been able to determine analytically.

The detailed calculation which leads to Eq. (2.3) can be found in Ref. [2, 7]. Since it relies on an Ansatz, numerical checks are required.

\subsection{Numerical checks}

We have implemented numerically the color dipole model in the form of a Monte Carlo event generator along the lines of Ref. [8], but with a modified BFKL kernel which simulates the effect of confinement through the cutoff function $\Theta$. We present in Fig. 2 the numerical results for $P_{n}$ for the following choice of this function:

$$
\Theta\left(\underline{r}_{1}, \underline{r}_{2} ; R_{\mathrm{IR}}\right)=\exp \left(-\frac{r_{1}^{2}+r_{2}^{2}}{2 R_{\mathrm{IR}}^{2}}\right),
$$

and setting the parameters to the values $\bar{\alpha} y=3, Q_{A}=50 / r_{0}, R_{\mathrm{IR}}=2 r_{0}$. (This Gaussian form was part of the Ansatz in Ref. [2]). Figure 2 shows that the asymptotics (2.3) with a constant $c$ in the range $0.5-0.6$ are fully consistent with the numerical calculations.

We are now aiming at investigating how the decay of $P_{n}$ at large $n$ depends on the infrared sector, i.e. how confinement is enforced onto the perturbative QCD calculation. Preliminary results seem to indicate that the dependence on the form of the cutoff function $\Theta$ is rather mild [7]. 


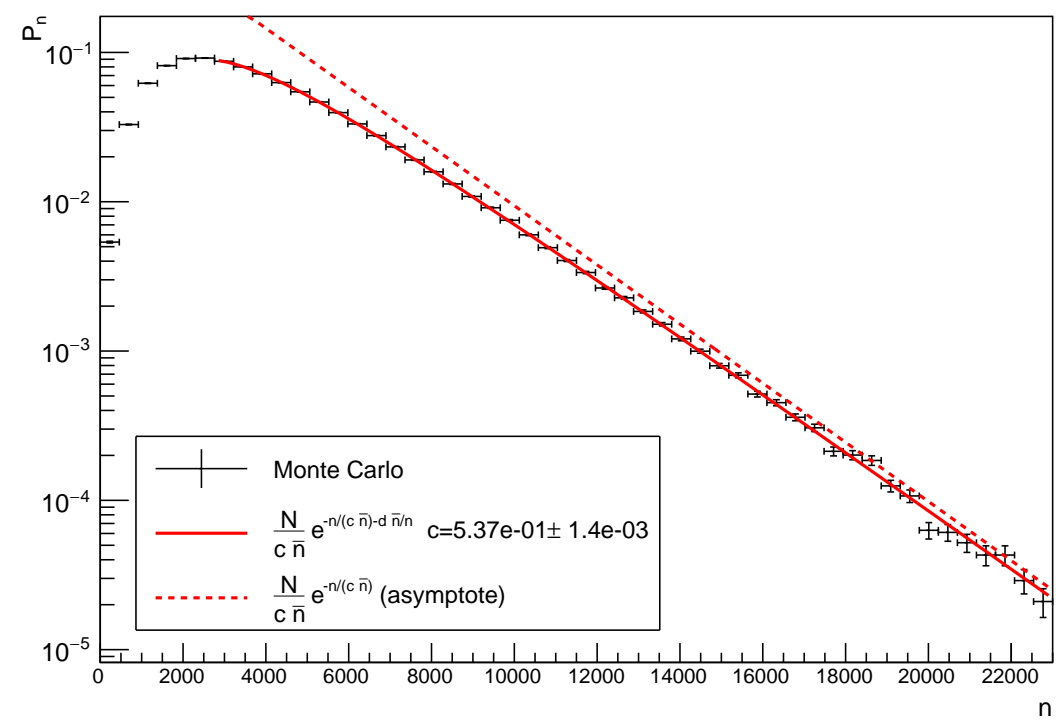

Figure 2: Points: Numerical data for $P_{n}$ for a particular cutoff function $\Theta$ and set of parameters (see the main text for details). Full line: Graph of the function $N /(c \bar{n}) e^{-n /(c \bar{n})-d \bar{n} / n}$, where the parameters $N, c$ and $d$ are determined from the least square method $\left(\chi^{2} /\right.$ d.o.f. $\simeq 38 / 41$ when $N \simeq 0.9, c \simeq 0.537$ and $d \simeq 0.71$ ). Note that we have added the arbitrary term $-d \times \bar{n} / n$ in the exponent to effectively take into account subasymptotic contributions which, so far, we have not been able to determine analytically. Dashed line: Large- $n$ asymptote.

\section{References}

[1] H. Mäntysaari and B. Schenke, Revealing proton shape fluctuations with incoherent diffraction at high energy, Phys. Rev. D 94, no. 3, 034042 (2016) [arXiv:1607.01711 [hep-ph]], and H. Mäntysaari, these proceedings.

[2] T. Liou, A. H. Mueller and S. Munier, Fluctuations of the multiplicity of produced particles in onium-nucleus collisions, Phys. Rev. D 95 no. 1, 014001 (2017) [arXiv:1608.00852 [hep-ph]].

[3] A. H. Mueller, Soft gluons in the infinite momentum wave function and the BFKL pomeron, Nucl. Phys. B 415, 373 (1994).

[4] For a review on hadronic and nuclear scattering at high energies, see e.g. Y. V. Kovchegov and E. Levin, Quantum Chromodynamics at High Energy, Cambridge Monogr. Math. Phys. 33 (2012).

[5] A. H. Mueller, Pictures of Particle Production in Proton-Nucleus Collisions, Acta Phys. Polon. B 47, 1955 (2016) [arXiv:1607.05623 [hep-ph]].

[6] G. P. Salam, Multiplicity distribution of color dipoles at small x, Nucl. Phys. B 449, 589 (1995) [hep-ph/9504284].

[7] L. Dominé, C. Lorcé, S. Munier and S. Pekar, in preparation.

[8] G. P. Salam, OEDIPUS: Onium evolution, dipole interaction and perturbative unitarization simulation, Comput. Phys. Commun. 105, 62 (1997) [hep-ph/9601220]. 